sensitivity. These chemical-genetic interaction (CGI) screens can be performed in human cell lines using a pooled lentiviral CRISPR-Cas9 approach to assess drug sensitivity/resistance of single-gene knockouts across the human genome. A targeted, rather than genomewide, library can enable scaling these screens across many drugs. CGI profiles can be derived from phenotypic screen readouts. These profiles are analogous to genetic interaction (GI) profiles, which represent sensitivity/resistance of gene knockouts to a second gene knockout rather than a drug. To computationally predict a drug's genetic target, we leverage the property that a drug's CGI profile will be similar to its target's GI profile. RESULTS/ANTICIPATED RESULTS: Five proof-of-principle screens will be conducted with compounds that have existing genome-wide profiles and wellcharacterized MOA. I will generate CGI profiles for these five compounds and identify genes that are drug-sensitizers or drugsuppressors. I will then evaluate whether targeted library screens can recapitulate the CGIs found in genome-wide screens. Finally, I will develop a computational tool to integrate these CGI profiles with GI profiles (derived from another project) to predict gene-level and bioprocess-level drug targets. These predictions (from both targeted and genome-wide profiles) will be benchmarked against a drug-target and drug-bioprocess standard. DISCUSSION/ SIGNIFICANCE OF FINDINGS: This work will develop a scalable, targeted chemical-genetic screen approach to discovering how putative therapeutics work. The targeted screen workflow provides a method for higher-throughput drug screening. The computational pipeline provides a powerful tool for exploring the MOA of uncharacterized drugs or repurposing FDA-approved drugs.

38081

A Whole Blood Signature of Neutrophil Expression and Adaptive Immune Downregulation Characterizes Sepsis Mortality

Giannini $\mathrm{HM}^{1,2}$, Cosgriff $\mathrm{CV}^{1,2}$, Lu XM ${ }^{1,2}$, Reilly $\mathrm{JR}^{2}$, Anderson $\mathrm{BJ}^{2}$, Jones $\mathrm{TK}^{2}$, Ittner $\mathrm{CAG}^{2}$, Weissman $\mathrm{AR}^{2}$, Agyeum $\mathrm{T}^{2}$, Dunn $\mathrm{T}^{2}$, Shashaty $\mathrm{M}^{2}$ and Meyer $\mathrm{NJ}^{2}$

${ }^{1}$ Institute for Bioinformatics, University of Pennsylvania; ${ }^{2} \mathrm{All}$ authors are from the Division of Pulmonary and Critical Care Medicine, Perelman School of Medicine, University of Pennsylvania, Philadelphia PA unless otherwise noted

ABSTRACT IMPACT: This work identifies host immune perturbations in sepsis mortality that suggests targets for a precision medicine paradigm in the host response to infection. OBJECTIVES/GOALS: To compare the early whole blood transcriptome during sepsis between 30-day survivors and non-survivors in the Intensive Care Unit (ICU), and to evaluate for pathway enrichment that might explain sepsis lethality. METHODS/STUDY POPULATION: We enrolled 162 sepsis patients in the first 24 hours of ICU admission, particularly targeting individuals requiring vasopressors. Peripheral whole blood was collected in PAXgene vacutainers. Isolated RNA was analyzed with Affymetrix Human Genome ST 2.0 microarray. Differential gene expression was performed with Bioconductor/R/ limma using log2 fold-change $+/-0.6$ as a threshold for differential expression, and a Benjamini-Hochberg adjusted p-value $<0.05$ to declare significance. Functional gene enrichment was performed using the Gene Ontology (GO) database with PANTHER overrepresentation test (Fisher's Exact) on all transcripts with adjusted p-value $<0.05$. Pathways analysis was performed with the Reactome Project using the raw fold change and significance data to identify dysregulated pathways. RESULTS/ANTICIPATED RESULTS: There were 58 non-survivors (36\% mortality). We identified 39 genes as differentially expressed between sepsis non-survivors and survivors; 31 were upregulated in non-survivors and 8 had reduced expression. Several of the most overexpressed transcripts are neutrophil-specific, including LCN, MPO, OLF4M4, DEFA3, and DEFA4. A functional gene overrepresentation test further supports this finding, as the most enriched gene ontologies were neutrophil-mediated killing, neutrophil cytotoxicity, neutrophil extravasation, and respiratory burst, all demonstrating higher than 10-fold enrichment and FDR $<0.02$. Pathway analysis of the peripheral blood transcriptome was notable for immune response derangement, specifically downregulation of both innate and adaptive immune pathways (FDR < 0.00001). DISCUSSION/SIGNIFICANCE OF FINDINGS: We identified increased expression of neutrophil-related genes in sepsis non-survivors, replicating candidates previously identified in pediatric sepsis mortality and ARDS. These immune perturbations in sepsis mortality may represent key targets for eventually employing precision medicine strategies in sepsis.

54443

\section{Pharmacogenomic Profiling of East and West African Populations}

Linsey Jackson ${ }^{1}$, Ruben Bonilla Guerrero ${ }^{2}$, Arjun Athreya ${ }^{1}$ and Lewis R. Roberts ${ }^{1}$

${ }^{1}$ Mayo Clinic; ${ }^{2}$ Admera Health

ABSTRACT IMPACT: Genomic variation likely plays a role in differences in rates of adverse reactions and efficacy for African populations, and this research will add to the understudied field of pharmacogenomics in African populations. OBJECTIVES/GOALS: We aim to characterize the frequency of variants in clinically relevant genes in East and West African populations to assess the prevalence of potential drug-gene interactions. METHODS/STUDY POPULATION: Our pilot study will consist of 100 Somali patients enrolled at Mayo Clinic Rochester and 100 Ghanaian patients recruited at Teaching Hospitals in Ghana. Germline DNA will be extracted from pre-existing blood samples. Sequencing will be performed using Admera Health's PGxOne Plus test, interrogating a panel of 62 genes. Variants will be reported along with the predicted response for a list of drugs. Differences between frequencies of variants in East and West African populations will be analyzed. We will look for correlations with reported adverse reaction rates. We will then compare our findings with allele frequency data from publicly available data bases. Additionally, we will analyze the flanking regions of the panel for novel variants, using machine learning to predict gene-drug interactions. RESULTS/ANTICIPATED RESULTS: African populations are known to have more genetic diversity than any other population. Additionally, only AfricanAmericans, African-Caribbeans from Barbados, Esan and Yoruba Nigerians, Gambians, Kenyans, and Sierra Leoneans are accounted for within the publicly available data bases most often used for variant studies. Thus, it is anticipated that we will find differences in the variant allele frequencies of our populations compared to European allele frequencies, and differences in frequencies between the East and West African populations. In the 200 base pair flanking regions that are sequenced in the assay along with the known variant regions, we may find novel previously unreported variants. DISCUSSION/ 